\title{
RESET
}

Recherches en sciences sociales sur Internet

\section{Les formes de l'exercice critique}

Une analyse comparée de la critique amateur et professionnelle sur un site Internet de cinéphiles

Forms and modes of critique. A comparative analysis of amateur and professional reviews on a website dedicated to films.

\section{Valérie Beaudouin et Dominique Pasquier}

\section{(2) OpenEdition}

\section{Journals}

Édition électronique

URL : http://journals.openedition.org/reset/684

DOI : $10.4000 /$ reset.684

ISSN : 2264-6221

Éditeur

Association Recherches en sciences sociales sur Internet

Référence électronique

Valérie Beaudouin et Dominique Pasquier, «Les formes de l'exercice critique », RESET [En ligne],

5 | 2016, mis en ligne le 30 juin 2016, consulté le 02 mai 2019. URL : http://journals.openedition.org/ reset/684; DOl : 10.4000/reset.684

Ce document a été généré automatiquement le 2 mai 2019.

(c) Association Recherches en sciences sociales sur Internet 


\section{Les formes de l'exercice critique}

Une analyse comparée de la critique amateur et professionnelle sur un site Internet de cinéphiles

Forms and modes of critique. A comparative analysis of amateur and professional reviews on a website dedicated to films.

Valérie Beaudouin et Dominique Pasquier

\section{Introduction ${ }^{1}$}

1 La critique professionnelle de cinéma est bien implantée dans les médias traditionnels et elle a été créditée d'une certaine influence sur le succès des films et dans la consolidation de nouveaux courants esthétiques (Debenedetti, 2006² ; Darré, 2006). Toutefois, comme dans d'autres secteurs culturels ${ }^{3}$, le pouvoir des critiques traditionnelles semble affaibli. Diverses raisons sont invoquées: pour les distributeurs, l'action des budgets de promotion semble plus efficace (Belvaux \& Marteaux, 2007 ; Beuscart \& Mellet, 2012), les jeunes générations lisent de moins en moins la presse (Donnat, 2009), l'indépendance des critiques est remise en question (Creton, 2000). Le développement massif d'évaluations de films postées en ligne par des cinéphiles amateurs pourrait bien constituer une concurrence menaçante (Béliard, 2014 pour les séries télévisées). Les chercheurs en marketing, qui ont été nombreux à s'y intéresser, estiment qu'on a là un nouvel instrument de prédiction du succès : le volume (et dans une moindre mesure la valence) des notes recueillies par un film serait un indicateur relativement fiable de ses performances commerciales à venir (Duan et al., 2008 ; Dellarocas, Zhang \& Awad, 2007 ; Larceneux, 2001 ; Liu, 2012). Quelques travaux se sont aussi intéressés au contenu même des critiques argumentées. Allard (2000) a étudié les échanges entre cinéphiles sur un forum consacré au cinéma à la fin des années 1990, en analysant les énoncés esthétiques sur les films. Elle distingue deux types d'intervenants, les « habitués cinéphiles » et les " novices simples ", et oppose les discours sur le modèle argumentatif aux avis purement subjectifs (du type "allez voir ce film»). Verboord (2014) a montré, sur un échantillon de critiques amateurs provenant de différentes plateformes de publication en ligne (forums, blogs, Webzines, médias traditionnels), que la forme de la critique dépend principalement 
du support de publication. Plus ce dernier est institutionnalisé et proche des médias traditionnels, plus les critiques centrées sur les qualités du film deviennent dominantes au détriment des critiques émotionnelles. La façon de rédiger dépendrait donc principalement du support médiatique, et la montée en visibilité de l'esthétique populaire serait due aux opportunités de publication dans des espaces non institutionnalisés.

2 Nous allons ici prolonger et compléter les travaux de ces deux derniers auteurs en comparant la critique professionnelle et la critique amateur. Cette comparaison peut être abordée sous plusieurs angles : le choix des films critiqués, la manière dont est pratiqué l'exercice critique, les arguments qui sont employés pour le mener. Mais aussi, comme l'a fait Allard, nous mettons en correspondance contenu des critiques et profil des auteurs, ce qui va nous permettre de montrer la grande hétérogénéité du monde de la critique amateur et les spécificités des élites de la contribution.

Notre analyse est fondée sur un corpus de 40000 critiques postées par 18000 contributeurs portant sur 141 films sortis en France en 2011. Le corpus a été prélevé sur un site français d'information sur le cinéma (appelé ici Viv@films) qui fournit des indicateurs chiffrés sur l'ancienneté de la participation des amateurs (date de première inscription) et sur l'intensité de leur engagement dans l'exercice critique (nombre de critiques déjà publiées).

\section{Méthodes}

\section{Délimitation d'un corpus de films}

Le corpus est constitué de l'ensemble des critiques formulées au sujet des films sortis en 2011 et encore à l'affiche en fin d'année. L'idée initiale aurait été d'avoir l'ensemble des films sortis en 2011. Mais, au moment de l'extraction en fin d'année, le moteur de recherche ne rendait plus accessible les films qui n'étaient plus à l'affiche. Notre principe de sélection privilégie donc les films sortis en fin d'année et les films qui sont restés longtemps à l'affiche (à la suite de leur succès critique ou en salle). Par ailleurs, au-delà de la date de décembre 2011 de nouvelles critiques ont pu être rédigées. La courbe de répartition des critiques en fonction de la date de sortie (figure 1) montre sur notre échantillon que $83 \%$ des critiques amateurs sont publiées avant la fin du premier mois suivant la sortie (à noter que $11 \%$ des critiques sont publiées avant la sortie officielle, indiquant ainsi que le film a été vu en avant-première ou que le spectateur est impatient de le voir). 17 \% des critiques sont donc publiées au-delà du premier mois. Ce pourcentage est plus élevé pour quelques films qui ont généré une grande activité critique sur un temps plus long. Pour The Tree of life sorti en mai 2011, on comptait 1582 critiques fin décembre 2011, au 30 juin 2014, 1 942, soit un tiers de plus. De même Intouchables, qui avait recueilli 4894 critiques fin 2011, en compte 6714 en juin 2014. L'activité de critique s'étend bien au-delà de la période de projection en salle, les DVD et autres modes de visionnage favorisant une longue traîne de distribution. 
Figure 1 : Délai de publication des critiques par rapport à la date de sortie du film

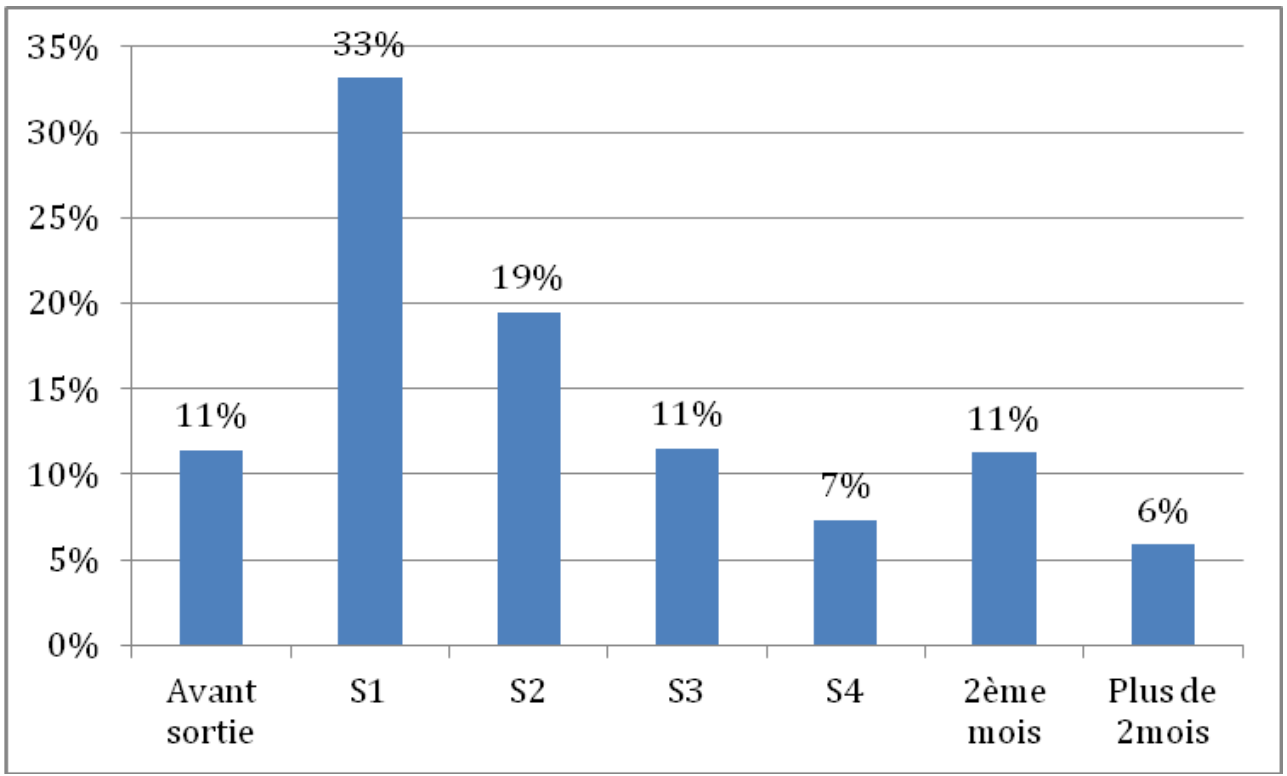

Champ : films sortis en 2011 et encore à l'affiche fin 2011 (N : 141).

Source : site français d'information sur le cinéma («Viv@films »).

5 La répartition du nombre de critiques par film (tableau 1) montre une grande inégalité dans l'activité de critique avec très peu de films qui reçoivent beaucoup de commentaires et un grand nombre qui en reçoit peu.

Tableau 1 : Nombre de films en fonction du nombre de critiques

\begin{tabular}{|l|l|}
\hline Nombre de critiques & Nombre de films \\
\hline Aucune critique & 6 \\
\hline Moins de 20 critiques & 33 \\
\hline De 20 à 100 critiques & 33 \\
\hline De 100 à 300 critiques & 32 \\
\hline Plus de 300 critiques & 37 \\
\hline (dont plus de 1000) & $(9)$ \\
\hline
\end{tabular}

Champ : films sortis en 2011 et encore à l'affiche fin 2011 (N : 141).

Source : site français d'information sur le cinéma («Viv@films »).

$6 \quad$ La distribution des critiques est corrélée aux résultats du box-office (tableau 2) : plus un film fait d'entrées, plus il reçoit de notes et d'avis critiques. La causalité fonctionne sans doute dans les deux sens. Quelques films s'écartent de ce modèle : ils ont pu faire l'objet de nombreuses critiques sans être aussi haut placés dans le box-office. C'est le cas de films primés et controversés au sein du public (The Tree of Life, Melancholia), et de films de genre comme Source code, Contagion, Real Steel. 
Tableau 2 : Comparaison entre le nombre de critiques spectateurs et les entrées du Box-office (films 2011)

\begin{tabular}{|c|c|c|c|}
\hline Titre & $\begin{array}{l}\text { Nombre de } \\
\text { critiques au } \\
31 / 12 / 2011\end{array}$ & $\begin{array}{l}\text { Entrées } \\
\text { Box-office }\end{array}$ & $\begin{array}{l}\text { Classement } \\
2011\end{array}$ \\
\hline Intouchables & 4894 & 19385300 & 1 \\
\hline Drive & 2805 & 1580623 & 30 \\
\hline $\begin{array}{l}\text { Les Aventures de Tintin: } \\
\text { Le Secret de la Licorne }\end{array}$ & 1951 & 5309485 & 4 \\
\hline The Tree of Life & 1582 & 872895 & 62 \\
\hline Polisse & 1567 & 2414418 & 19 \\
\hline The Artist & 1198 & 3064827 & 9 \\
\hline $\begin{array}{l}\text { Twilight - Chapitre } 4 \text { : } \\
\text { Révélation 1ère partie }\end{array}$ & 1168 & 3638210 & 7 \\
\hline Minuit à Paris & 1130 & 1739215 & 27 \\
\hline Source Code & 1115 & 710729 & 80 \\
\hline $\begin{array}{l}\text { Mission : Impossible - } \\
\text { Protocole fantôme }\end{array}$ & 897 & 2415036 & 18 \\
\hline Time Out & 832 & 1099016 & 47 \\
\hline Contagion & 791 & 686151 & 83 \\
\hline Melancholia & 724 & 409246 & 122 \\
\hline Real Steel & 675 & 1101070 & 46 \\
\hline Comment tuer son Boss? & 624 & 814141 & 67 \\
\hline La Guerre est déclarée & 621 & 837880 & 65 \\
\hline Destination Finale 5 & 574 & 956967 & 57 \\
\hline Hugo Cabret & 563 & 1285647 & 40 \\
\hline La Piel que Habito & 543 & 733758 & 76 \\
\hline Cars 2 & 535 & 2975790 & 11 \\
\hline
\end{tabular}

Note : le classement dans la dernière colonne est fait sur l'ensemble des films sortis en 2011 et non sur les 141 de notre corpus. Les données sur le nombre d'entrées en salles proviennent de JP's Box Office.

Champ : films sortis en 2011.

Source : site français d'information sur le cinéma («Viv@films ») ; JP’s Box Office.

\section{Extraction des données présentes sur le site à plusieurs niveaux}

7 La démarche adoptée a été la suivante : en partant de chacun des 141 films, toute une série de données ont été extraites de la plateforme, à savoir les caractéristiques du film, les critiques presse, les critiques spectateur et les caractéristiques disponibles sur les auteurs des critiques. 
8 Les caractéristiques du film recueillies sont le titre, son ou ses réalisateurs, les comédiens principaux, la nationalité, le genre et la date de sortie. Le genre est attribué par Viv@films et comprend d'une à trois valeurs. Les films sont également caractérisés par des indicateurs synthétiques liés à la critique spectateur et presse : nombre de notes et de critiques spectateur, note moyenne (sur cinq), effectifs sur chaque note (étoile), nombre de critiques presse et note moyenne. En revanche nous n'avons pas extrait les données concernant les prix obtenus, les budgets et le box-office, qui, à cette époque, n'étaient pas disponibles sur la plateforme de manière systématique. Une table, composante d'une base de données relationnelle, comprenant tous ces traits de description des films a ainsi été constituée.

9 Pour les critiques des spectateurs, une autre table a été créée. Elle contient la critique rédigée avec la note associée, le film dont il est question, l'auteur de la critique, mais aussi la date et l'heure de publication de la critique.

De la même manière, une table regroupe les critiques de la presse (le plus souvent il s'agit d'une courte citation de l'article sélectionné), une note (attribuée par le site à la lecture de la critique), le nom du média et du journaliste.

Enfin, une dernière table consigne les informations disponibles sur les auteurs des critiques spectateurs : leur date d'inscription sur le site, le nombre de critiques rédigées depuis leur date d'inscription, leur nombre d'abonnés et leur appartenance ou non au Club. Le Club (qui compte un peu moins de 500 membres) a été créé par le site en 2008, avec des principes assez différents de celui des top reviewers d'Amazon (Beaudouin \& Pasquier, 2014) 4 . Ce n'est pas le volume de contribution qui donne accès au Club, ni le nombre de votes «utiles» - la possibilité de noter «utile» une critique n'existait d'ailleurs pas avant 2013.

12 Les fiches profils étant très peu remplies, nous n'avons malheureusement pas pu utiliser les données de genre ou d'âge. C'est évidemment la principale limite de ce type d'approche qui part de l'activité et des contenus visibles sur les plateformes sans pouvoir remonter aux caractéristiques et motivations des individus. On voit bien tout l'intérêt qu'il pourrait y avoir à compléter cette démarche par une enquête auprès des rédacteurs eux-mêmes, comme a pu le faire Naulin (2014) dans son enquête sur les blogueurs culinaires.

$13 \mathrm{Au}$ final, sur un corpus de 140 films, nous avons extrait 40000 critiques spectateurs, rédigées par 18000 spectateurs différents et 2300 critiques presse.

\section{Enrichissement des données}

À partir de ces données extraites de la plateforme, nous avons construit des variables et indicateurs complémentaires pour enrichir la description, sans avoir recours à des savoirs externes sur le film.

Pour les films, nous avons retravaillé la typologie des genres proposée par Viv@Films (de 1 à 3 genres par film) pour affecter un seul genre à chaque film et réduire le nombre de catégories. Finalement, les films relèvent des catégories : Documentaire, Drame, Comédie dramatique, Comédie, Animation, Action-Thriller-SF. Cette dernière est un regroupement que nous avons fait en raison de la proximité de leurs caractéristiques. Nous avons privilégié le premier genre donné par la plateforme, supprimé des genres caractérisant le public («Famille») et remplacé par un genre plus fréquent les genres rares («Biopic », 
«Guerre » ...). Nous avons aussi construit un indicateur de préférence sur les films qui permet d'appréhender les écarts de notation entre les spectateurs et la presse. Nous avons réparti les films en trois catégories : les films nettement préférés par les amateurs (écart de note $>0,7$ ), les films nettement préférés par la presse (écart de note $<0,3$ ) et les autres. Les deux catégories extrêmes regroupent chacune un quart des films. Une variable permet de répartir en quatre groupes de taille équivalente les films en fonction du nombre de notes reçues : de 0 à 58 notes, de 59 à 441 notes, de 442 à 1254 et plus de 1255 . Ces nouvelles variables viennent s'ajouter aux premières.

Pour les auteurs de critiques, des indicateurs complémentaires ont été construits: l'ancienneté d'inscription sur le site (moins de 3 mois, 3 mois à un an et demi, un an et demi à 4 ans, plus de 4 ans), le nombre de critiques rédigées depuis l'inscription (1 critique, 2 à 12 critiques, 13 à 50 , plus de 50 critiques), le nombre de critiques rédigées sur les films 2011 (1 critique, 2, 3 à 5, 6 à 11, plus de 11 critiques).

Ceci nous permet d'observer la répartition du nombre de contributeurs et du nombre de critiques en fonction de l'intensité de l'activité. $10 \%$ des contributeurs sur les films de 2011 avaient déjà rédigé plus de 50 critiques depuis leur inscription sur la plateforme et ils ont produit plus d'un tiers des contributions. Ceux qui n'ont écrit qu'une fois représentent un tiers des contributeurs, mais seulement $15 \%$ des textes (tableau 3).

Tableau 3 : Répartition des contributeurs et des critiques selon le nombre de critiques rédigées depuis l'inscription

\begin{tabular}{|l|l|l|l|l|}
\hline Depuis l'inscription & $\begin{array}{l}\text { Nombre de } \\
\text { contributeurs }\end{array}$ & $\begin{array}{l}\text { \% de } \\
\text { contributeurs }\end{array}$ & $\begin{array}{l}\text { Nombre de } \\
\text { critiques }\end{array}$ & $\begin{array}{l}\text { de } \\
\text { critiques }\end{array}$ \\
\hline 1 critique & 5857 & 32 & 5857 & 15 \\
\hline 2 à 12 critiques & 7829 & 43 & 12392 & 31 \\
\hline 13 à 50 critiques & 2711 & 15 & 7904 & 20 \\
\hline Plus de 50 critiques & 1780 & 10 & 13321 & 34 \\
\hline Total & 18177 & 100 & 39474 & 100 \\
\hline
\end{tabular}

Champ : films sortis en 2011 et encore à l'affiche fin 2011 ( $N$ : 141).

Source : site français d'information sur le cinéma («Viv@films »).

18 Si l'on se restreint à l'activité critique concernant les films de 2011, les disparités sont renforcées ; $69 \%$ des contributeurs n'ont écrit qu'une seule critique et $3 \%$ plus de 11 critiques qui couvrent $24 \%$ du corpus.

Les critiques elles-mêmes sont d'une grande richesse, et régulièrement nous avons lu et relu des échantillons de critiques pour confirmer, affiner des hypothèses ou pour en faire émerger de nouvelles. Il nous a paru cependant nécessaire de pouvoir résumer les critiques par quelques indicateurs. Le délai de rédaction de la critique par rapport à la

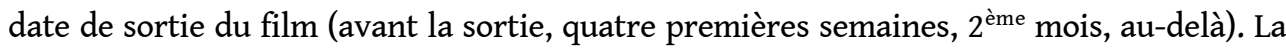
longueur de la critique, comptée en nombre de mots, a été organisée en 4 catégories, selon les quartiles, des plus courtes au plus longues (de 0 à 25 mots, de 26 à 45 , de 46 à 92 et de 93 à 900 mots). Pour appréhender les contenus des critiques de manière systématique, nous avons eu recours aux méthodes de text mining qui permettent de produire des typologies de textes à partir des cooccurrences des mots employés (logiciels 
Alceste et Iramuteq). Sont regroupées ensemble les critiques qui emploient le même type de vocabulaire.

Le corpus de critiques est marqué par les mécanismes propres à la contribution sur internet : très peu d'individus qui interviennent beaucoup et une grande masse de très faibles contributeurs (distribution en loi de puissance). A cela, s'ajoutent des mécanismes de boule de neige et de concentration de l'attention qui expliquent de grandes disparités dans le nombre de critiques par film (5000 pour Intouchables, alors qu'un quart des films en a reçu moins de 20). Comme les méthodes de text mining partent des corpus pour faire émerger des catégories, il nous a fallu échantillonner pour atténuer les effets de cette distribution en loi de puissance (sinon nous aurions obtenu une grande classe liée à Intouchables opposée à toutes les autres). Nous avons pris pour option de garder au maximum les 400 premières critiques produites à partir de la date de sortie du film, soit un corpus de 17280 critiques (un peu moins de la moitié de nos critiques).

21 Ces données enrichies sont soumises à des traitements statistiques : tris à plat et croisés avec tests de significativité.

\section{L'amateur face à la critique professionnelle}

Sur le site que nous avons étudié, les critiques professionnelle et amateur cohabitent à même niveau d'accès. Les évaluations professionnelles apparaissent dans une rubrique " presse ", et sont puisées dans une trentaine de titres de la presse quotidienne nationale et de la presse magazine. L'article sur un film est analysé par le service éditorial du site qui choisit un extrait jugé représentatif et attribue une note à la place du journaliste si ce dernier ne l'a pas fait. Une note moyenne presse est calculée à partir de l'ensemble de ces notes ; par un clic il est possible de descendre au niveau des notes attribuées par chacun des supports presse. Un autre clic permet d'accéder à l'intégralité de l'article sur le site web du titre. En clair, ce qui est le plus directement accessible sur ce site est une opération de retraduction qui présente des caractéristiques bien particulières : une note moyenne - qui tend donc à écraser les divergences entre journalistes -, fondée sur un petit nombre d'articles (tous les films ne font pas l'objet d'une critique dans les supports couverts). Les évaluations des internautes apparaissent directement sous celles de la presse dans une rubrique dénommée "spectateurs». Il s'agit aussi d'une note moyenne fondée sur l'ensemble des notes attribuées par les internautes, avec une indication chiffrée du nombre de notes (qui peut se monter à plusieurs dizaines de milliers pour les films les plus évalués) et du nombre de critiques argumentées (dans un rapport de 1 à 4 par rapport aux notes). Plusieurs clics successifs permettent d'accéder au texte intégral d'une critique. Nous avons donc deux indicateurs qui ne sont pas du tout construits de la même façon mais qui tous deux écrasent la diversité des jugements. En dépit de ces modes de construction différents, leur mise en équivalence sur le site nous incitera à comparer les notes des spectateurs et les notes de la presse, d'autant plus que les critiques font souvent référence à ces notes moyennes.

Une première question se pose. Doit-on qualifier de « critiques » les textes des amateurs? Dans un article de 2010, Verboord les appelle lay critics par opposition à expert critics, mais la plupart des chercheurs emploient le couple reviews / ratings, ou en français avis / notes. Dans le cas étudié ici, le terme de critique s'impose. Tout d'abord parce que c'est le terme employé par le site qui place ces textes au même niveau d'accès que les critiques presse. Les responsables de Viv@films ont aussi rédigé une « charte des critiques » qui donne des 
indications très précises sur le format et le type de critique qui est attendu ${ }^{5}$. Ce qu'on propose aux internautes est bel et bien d'écrire une « critique ».

C'est aussi ce que vivent les contributeurs. Une lecture attentive des textes montre vite qu'ils prennent l'exercice très au sérieux: " $D$ 'habitude avant de faire une critique je revois le film histoire de pas dire n'importe quoi», «je vais me faire de nombreux ennemis avec cette critique mais je rappelle que c'est le film et non pas l'histoire vraie que je juge. Et encore je suis très généreux » (il a noté 2 sur 5). Cela apparaît aussi au niveau de la forme - orthographe, vocabulaire -, mais surtout, la plupart des rédacteurs semblent animés par l'idée que leur critique aura du poids. Ils cherchent à convaincre et au moment de donner une note au film, ils sont mus par un sentiment de responsabilité : " J'ai longtemps hésité entre 4 et 4.5 étoiles !!! Comme je ne me suis pas ennuyé un seul instant (ce qui arrive fréquemment avec les block américains) et comme l'acteur jake Gyllenhaal est très bon, j'ai penché vers le 4.5. ». Enfin, les allusions aux autres critiques des internautes et aux critiques de la presse sont fréquentes. On écrit en relation avec d'autres en ayant lu ce que disent les autres. Critiquer c'est s'engager : défendre un film, convaincre des spectateurs, éventuellement contrer les jugements experts.

En plus des différences explicitées que nous venons de présenter, les critiques amateurs se distinguent de celles de la presse dans trois domaines : le choix des films, les manières de noter, et le recours à un registre émotionnel dans l'exercice critique.

\section{Le choix des films}

Si l'on classe les films par nombre croissant de critiques reçues (figure 2), la première moitié des films recueille $30 \%$ des critiques presse mais seulement $5 \%$ des critiques spectateurs. Inversement, la moitié des critiques amateur porte sur $10 \%$ des films seulement (qui ne recueillent que $16 \%$ de la critique presse). Or ces films, qui concentrent l'attention des spectateurs, font partie de ceux qui ont fait le plus grand nombre d'entrées en salle. L'attention de la presse se distribue plus équitablement entre les différents films que celle des spectateurs, qui se focalise plus souvent sur les succès du box-office. 
Figure 2 : Courbe cumulée des critiques amateurs et professionnelles par film

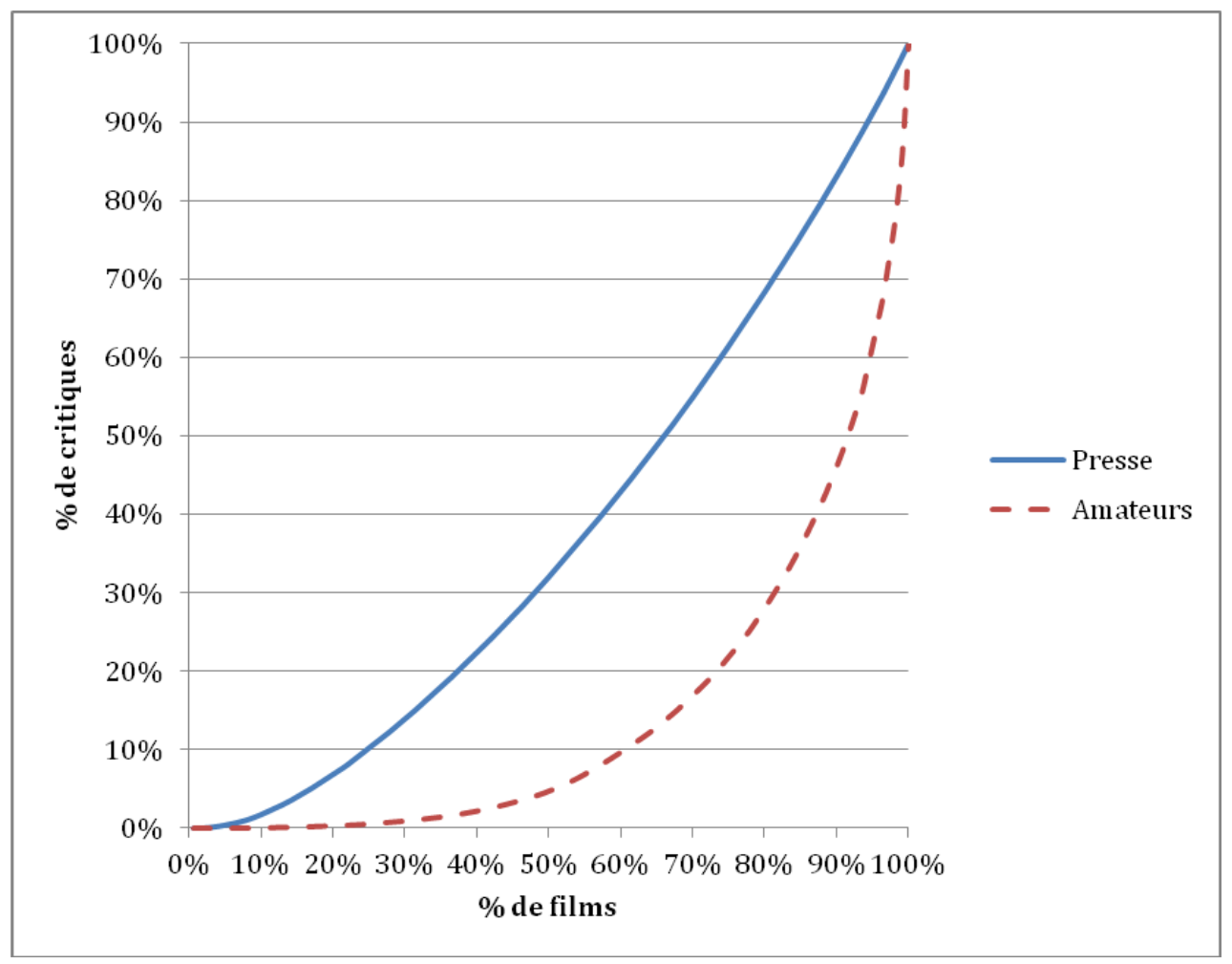

Champ : films sortis en 2011 et encore à l'affiche fin 2011 (N : 141).

Source : site français d'information sur le cinéma («Viv@films »).

L'étude de la répartition du nombre de critiques par genre (tableau 5) montre que la critique professionnelle couvre d'une manière plus homogène l'ensemble des genres, même si elle tend à privilégier les genres traditionnellement dominants : le drame plutôt que la comédie, les genres nobles plutôt que les «mauvais genres» (comme sciencefiction, action, horreur).

Les écarts avec la critique amateur sont sensibles. Il faut souligner en premier lieu la surreprésentation très nette chez les amateurs de critiques de films "Action-thrillerScience-fiction » : $29 \%$ des critiques portent sur des films relevant de ces genres, contre $14 \%$ des critiques presse alors même que ces genres représentent $13 \%$ des films. Le tableau 5 montre aussi chez les amateurs une nette préférence pour les comédies ( $25 \%$ au lieu de $16 \%$ pour la critique presse). Inversement, les documentaires et les drames suscitent nettement moins d'intérêt : $25 \%$ des critiques amateurs portent sur des drames, contre $38 \%$ des critiques presse et $1 \%$ sur des documentaires, contre $10 \%$ pour la presse.

Tableau 4 : Répartition des critiques presse et spectateurs selon les genres de films (\%)

\begin{tabular}{|l|l|l|l|}
\hline & Films & $\begin{array}{l}\text { Critiques } \\
\text { presse }\end{array}$ & $\begin{array}{l}\text { Critiques } \\
\text { spectateurs }\end{array}$ \\
\hline Drame & 32 & 38 & 25 \\
\hline Comédie dramatique & 10 & 13 & 10 \\
\hline Comédie & 18 & 16 & 25 \\
\hline
\end{tabular}




\begin{tabular}{|l|l|l|l|}
\hline Animation & 12 & 9 & 11 \\
\hline Action Thriller SF & 13 & 14 & 29 \\
\hline Documentaire & 15 & 10 & 1 \\
\hline Total (\%) & 100 & 100 & 100 \\
\hline Total effectifs & 140 & 2324 & 39498 \\
\hline
\end{tabular}

Champ : films sortis en 2011 et encore à l'affiche fin 2011 (N : 141).

Source : site français d'information sur le cinéma («Viv@films »).

\section{Les manières de noter}

Dans l'ensemble, les internautes attribuent aux films des notes plus élevées que la presse. Ce biais positif dans les évaluations profanes a été relevé par de nombreux chercheurs dans des secteurs très différents (Mayzlin \& Chevalier, 2003 ; Pasquier, 2014). Dans notre corpus, la moyenne des notes amateurs est de 3,39 et celle des critiques professionnels de 3,1. En dépit d'une note moyenne assez proche, la différence est statistiquement significative. En termes de distribution sur nos films, il apparaît que les notes des critiques professionnels (telles qu'elles sont attribuées par le site) sont plus modérées que celles des amateurs, évitant les notes trop basses mais aussi les notes très élevées (tableau 6). Les amateurs se laissent emporter plus facilement par leurs émotions (rejet ou enthousiasme).

Tableau 5 : Répartition des notes selon les catégories de critiques (amateurs vs professionnels) (\%)

\begin{tabular}{|l|l|l|l|l|l|l|}
\hline & $\begin{array}{l}\mathbf{0 , 5} \\
\text { et 1 }\end{array}$ & $\mathbf{2}^{*}$ & $\mathbf{3}^{*}$ & $\mathbf{4}^{*}$ & $\mathbf{5}^{*}$ & Total \\
\hline Spectateurs & 16 & 15 & 25 & 27 & 17 & 100 \\
\hline Presse & 9 & 17 & 28 & 32 & 14 & 100 \\
\hline
\end{tabular}

Champ : films sortis en 2011 et encore à l'affiche fin 2011 ( $N$ : 140).

Source : site français d'information sur le cinéma («Viv@films »). 
Figure 3 : Répartition des critiques selon les notes (\%)

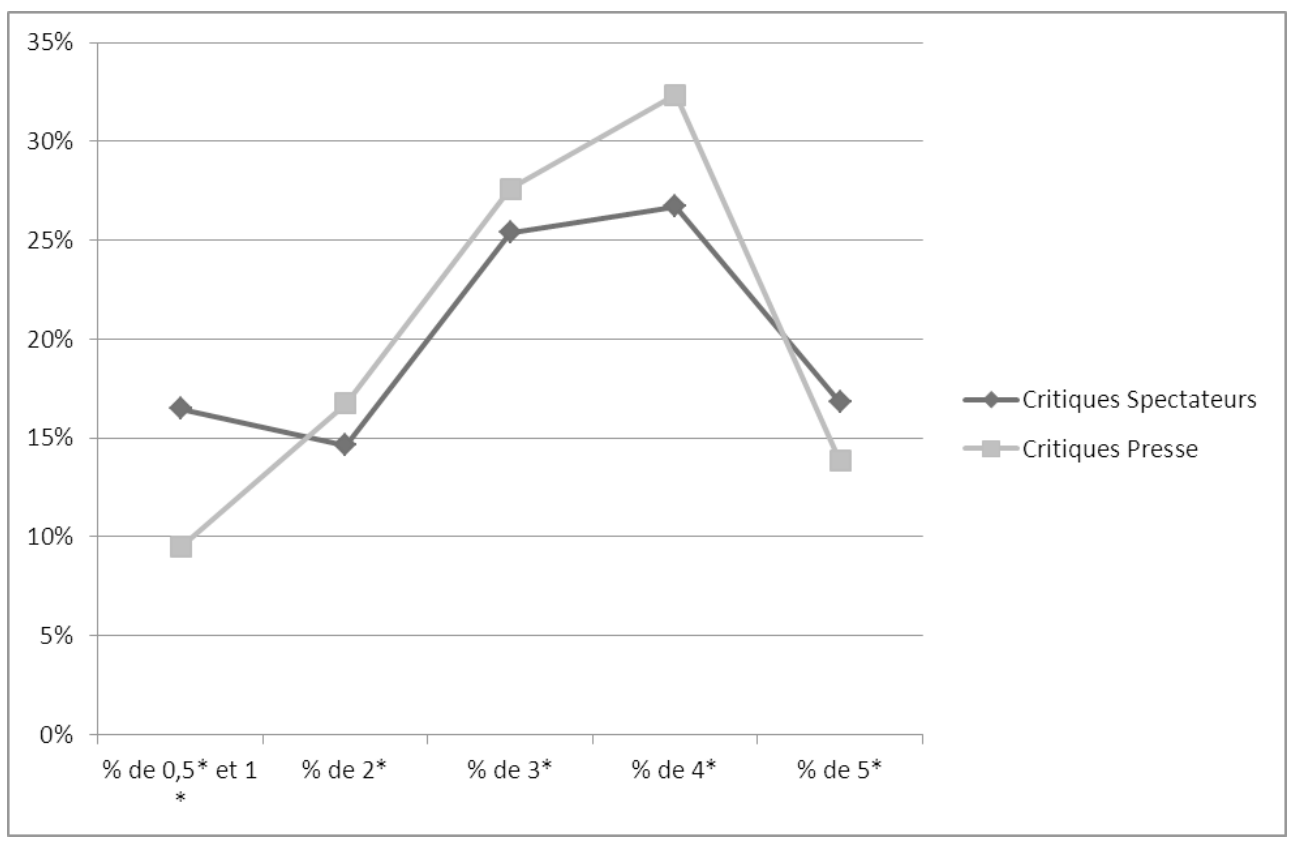

Lecture : en moyenne, la note de $5^{\star}$ est attribuée par $17 \%$ des spectateurs. On note que la critique professionnelle (telle qu'elle est construite par Viv@films) est plus mesurée (moins de notes très basses et très hautes) que la critique amateur.

Champ : films sortis en 2011 et encore à l'affiche fin 2011 (N : 141).

Source : site français d'information sur le cinéma («Viv@films »).

Les décalages entre la notation presse et amateur peuvent être appréhendés à travers l'écart entre la note moyenne des deux catégories. Nous avons distingué les films préférés par la presse d'un côté et ceux que préfèrent les spectateurs de l'autre. Les écarts de notation sont largement liés à la question des genres. Les genres privilégiés par la presse sont aussi les genres pour lesquels la note presse est plus élevée que la note spectateur : drames, documentaires, etc. Inversement, comédies et films d'animation sont préférés par les amateurs: ils font à la fois l'objet de plus de critiques et de meilleures notes (figure 4). 
Figure 4 : Répartition par genre des critiques selon l'appréciation des films (\%)

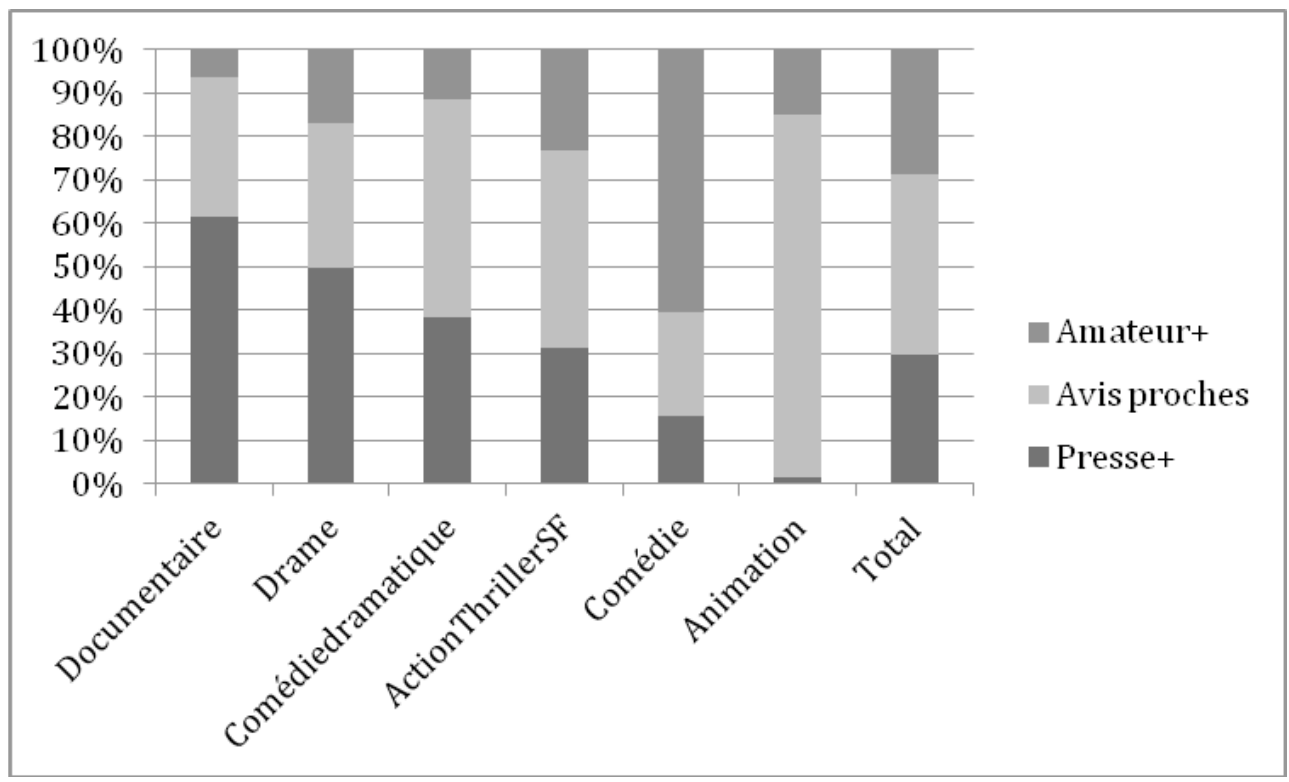

Lecture : $60 \%$ des critiques de documentaires portent sur des films qui plaisent bien plus à la presse qu'aux amateurs (un film plaît plus à la presse si l'écart entre la moyenne amateur et presse est inférieure à -0,7).

Champ : films sortis en 2011 et encore à l'affiche fin 2011 ( N : 141).

Source : site français d'information sur le cinéma («Viv@films »).

Une analyse plus attentive des critiques des films qui suscitent un fort désaccord de notation entre presse et amateurs permet d'identifier deux cas de figure.

Dans le premier cas, il s'agit de films que les internautes ont « sur-notés » par rapport à la presse. Il est intéressant de constater que cette catégorie peut regrouper des films de genres assez différents pour lesquels les amateurs se mobilisent au nom d'un même principe : ils ont éprouvé des émotions fortes en les voyant. Il peut s'agir de comédies, de drames ou de films d'action. Ils ont en commun d'avoir été peu appréciés de la presse qui en a critiqué les ficelles "grossières » et les recettes «tire-larmes ». En réaction, les internautes dénoncent la froideur de la critique professionnelle et revendiquent haut et fort les émotions comme une dimension fondamentale de l'expérience cinématographique. Intouchables (4,6 de note moyenne chez les amateurs contre 3,7 pour la presse) est un exemple paradigmatique. La note maximale de 5 , que plus de $60 \%$ des 4 516 internautes ayant écrit une critique du film ont attribué à Intouchables en 2011, est ainsi brandie comme le serait un drapeau : «je voulais voter 10 étoiles hélas on ne peut en mettre seulement $5 \wedge^{\wedge} \wedge^{\prime}$ ", « 5 étoiles, s'il y en avait eu plus, je les aurais mises....ce film, c'est du pur bonheur », « Les 5 étoiles coulent de source !!! ». Ces critiques se mobilisent clairement contre les approches considérées comme trop analytiques ou cérébrales de la presse :

Merveilleux, il n'y a pas d'autres mots! Après avoir passé toute l'année avec au moins deux films par semaine dans les jambes, c'est sans hésitation que je décerne à "Intouchables" le prix du film de l'année ! Omar révélé, François ressuscité. A voir, revoir et rerevoir! Des fous rires tout le long et même quelques larmes viennent ponctuer de vrais moments d'émotion. Quand à ceux qui viennent parler de clichés et d'un scénario trop simplet, c'est uniquement pour jouer la carte de l'anticonformisme et se détacher de l'émulation collective que j'ai pu voir dans la 
salle et dans la majorité des commentaires. Merci pour cette merveille en tout cas. ( Intouchables, note 5)

33 Au sujet de ce type de film, les attaques contre les journalistes qui « se permettent » de critiquer peuvent être très directes : « Par contre je ne félicite pas la critique de Télérama qui se veut être élitiste dans ses opinions et qui pour une fois (encore ?) est passé à côté du film. »

Des drames comme La couleur des sentiments (4,1 de note moyenne chez les amateurs contre 2,5 pour la presse) ou dans une moindre mesure Polisse $(4,1$ contre 3,7$)$ provoquent des réactions scandalisées à l'égard des critiques professionnels qui se sont montrés insensibles à l'intensité des histoires vécues par les personnages.

Voilà encore un film qui me conforte dans l'idée de ne jamais regarder les critiques presse, désolé m'sieurs dames. Film splendide à consommer sans modération, les yeux et le cœur grand ouvert. Presque toute la salle dont moi était en pleurs. ( $\mathrm{La}$ couleur des sentiments, note 5).

Dans la catégorie des films d'action, Forces spéciales engendre un décalage particulièrement important entre les moyennes attribuées par la presse $(1,2)$ et par les amateurs $(3,9)$. C'est un film de guerre français qui a déclenché les foudres de l'ensemble de la critique professionnelle («nanar », « navet ", " ridicule ») et par retour suscité un véritable contre-feu chez les amateurs : « la presse est incompréhensible », " la presse ne doit pas avoir vu le même film que moi ", « un très beau film qui ne mérite en rien les critiques assassines de la presse qui n'y a vraisemblablement rien compris », " encore un coup de journaleux aigris, poussiéreux et soi disant bien pensant », « les critiques feraient mieux de la fermer, quand on voit les daubes que vous soutenez ", " restez dans votre belle tour d'ivoire de cinéma pseudo intello », etc.

On pourrait multiplier les exemples. L'important est de comprendre que ces mobilisations au nom de la place des émotions dans l'expérience des films sont formulées sur le ton d'une véritable indignation morale. Un film qui a fait pleurer ou rire, et surtout les deux à la fois, ne doit pas être soumis au jugement froid du professionnel.

37 Taxés d'insensibilité, les auteurs de critiques professionnelles sont aussi soupçonnés de complaisance à l'égard des valeurs déjà reconnues, comme s'ils défendaient plus le milieu du cinéma que l'intérêt des spectateurs. On est là dans un cas de figure inverse du précédent puisqu'il s'agit de films sous-notés par les spectateurs comparativement à la presse. Cependant, les raisons du décalage ne sont pas fondamentalement différentes, puisque c'est au nom d'une expérience émotionnelle - cette fois-ci insatisfaisante - que ces films sont contestés par les amateurs. La collusion entre les critiques de la presse et les jurys des prix est au fondement de la plupart des critiques amateurs au sujet de ces films, et «l'ennui » le nouveau chef d'accusation. Les films de cette catégorie ont en commun d'avoir reçu des récompenses importantes (notamment à Cannes) ou/et d'avoir été réalisés par des réalisateurs très reconnus. Du festival de Cannes de 2011 sont ainsi contestés la palme d'or, The Tree of life (3,5 note presse contre 2,7 amateurs), le grand prix, Le gamin au vélo $(4,2$ contre 3,4$)$, le prix de la mise en scène Drive $(4,4$ contre 3,8$)$ et le prix d'interprétation féminine, Melancholia (4,1 contre 3,5). Mais ce sont aussi des réalisateurs célèbres comme Moretti pour Habemus papam (4 contre 3,4$)$ ou Woody Allen pour Minuit à Paris $(4,2$ contre 3,6$)$. Tous ces films ont en commun d'avoir reçu une très bonne critique presse alors que les spectateurs disent s'être ennuyés, n'avoir pas compris où les réalisateurs voulaient en venir, et avoir constaté que de nombreux spectateurs étaient dans le même cas qu'eux puisqu'ils quittaient la salle : 
Un film creux loin, très loin derrière les avis des critiques de Cannes. pour pseudo intellos uniquement, juste histoire pour ceux qui veulent dire que j'ai vu un Lars Von Trier. je ne comprends pas le hourra de la presse. (Melancholia)

C'est chiant au possible, tellement chiant que vous risquez de quitter la salle avant la fin ce que j'ai fait. (The Tree of life)

Je ne comprends pas les 5/5 en série de la presse », "y a de quoi s'interroger sur les critiques professionnels ", "que dire sur ce film qui a été encensé par la critique et les spectateurs... (Drive) l'ensemble des sources de désaccord entre la presse et les spectateurs. Certains décalages peuvent trouver leurs racines ailleurs: les films d'animation par exemple sont dans l'ensemble beaucoup plus défendus par les amateurs que par la presse, comme le sont aussi les films de science-fiction ou d'horreur. Visiblement, ces genres délaissés par la critique font au contraire partie des films qui mobilisent les amateurs. À l'inverse, des films que la presse a appréciés en raison de leur audace peuvent choquer les critiques amateurs. C'est le cas de La guerre est déclarée (de Valerie Donzelli : presse 4,4, spectateurs 3,7) ou de Shame (de Steve Mac Queen : 4 contre 3,4), deux films qui ont reçu de nombreux prix et une excellente critique presse. Les spectateurs ont détesté que le premier mette en scène le cancer de l'enfant de la réalisatrice, et ils ont été outrés des scènes sexuelles trop crues du second.

\section{L'utilisation du registre émotionnel}

Enfin, il existe un registre critique chez les amateurs dont on ne trouve pas d'équivalent dans la presse : une critique émotionnelle centrée sur la réception, et les effets du film sur le spectateur.

Une analyse des contenus textuels des critiques de notre corpus fait en effet apparaître deux modèles fortement contrastés avec, d'un côté des critiques centrées sur le contenu et la forme du film et de l'autre des critiques centrées sur la réception, sur ce que le film fait au spectateur. Ces deux modèles critiques sont très proches de ceux identifiés par une série de travaux sur les contenus de la critique amateur en ligne (Allard, 2000 ; Legallois \& Poudat, 2008; Verboord, 2014). Ces recherches s'accordent à identifier deux pôles de jugement, l'un fondé sur les qualités esthétiques du bien, l'autre sur le ressenti émotionnel en tant que consommateur, avec des terminologies qui varient selon les auteurs mais qui reprennent toutes une opposition identifiée par Bourdieu entre disposition esthétique qui permet de s'approprier l'œuvre « dans sa forme plutôt que dans sa fonction", et esthétique populaire, qui «implique la subordination de la forme à la fonction » (Bourdieu, 1979 : 33).

Les outils de fouille de texte utilisés sur notre corpus de critiques amateur (Alceste et Iramuteq) distinguent deux grandes classes de critiques, de taille équivalente, sur la base des cooccurrences de vocabulaire. Par construction, chaque classe est la plus homogène possible en termes de vocabulaire utilisé et se distingue fortement de l'autre classe. 
La première rassemble des critiques longues avec une grande richesse de vocabulaire (le taux d'hapax - mots n'apparaissant qu'une seule fois - est de $38 \%$ alors qu'il n'est que de $24 \%$ dans l'autre classe). Le vocabulaire spécifique de la classe, à savoir les mots qui sont significativement plus présents dans cette classe, est particulièrement riche et permet d'identifier les caractéristiques de ces critiques :

réalisateur, vie, mise en scène, homme, spectateur, femme, sujet, cinéma, personnage, plan, montrer, sentir, dernier, filmer, sembler, amour, force, monde, vivre, prendre, image, jeune, côté, cinéaste, grand, jour, beauté, politique, devenir, humain, regard, rendre, travail, laisser, réalité, parler, lumière, comprendre, œuvre, interprétation, donner, rester, manière, question, venir, drame, mère, art, fois, premier, partie, propos, propre, documentaire, minute, corps, façon, difficile, temps, doute, social, noir, canne, face, agir, com, raconter, caméra, séquence, auteur, fond, époque, récit, sortir, travers, long...

Les critiques portent sur les films en tant que processus artistique et en tant que bien culturel. Une attention est accordée à la fabrique du film (mise en scène, filmer, montrer, raconter), à ses ingrédients (personnages, sujet, plan, séquence, genre...), au monde de l'art (réalisateur, spectateur, comédiens...). Par ailleurs, ce sont des critiques qui sont centrées sur le contenu du film et sur sa fonction référentielle (de quoi parle le film).

Inversement, dans la seconde classe, les critiques sont plus courtes, mobilisent un vocabulaire plus restreint, comme en témoigne le plus faible nombre de mots spécifiques :

sympa, super, rire, adorer, dessin animé, gag, rigoler, drôle, marrant, décevoir, action, divertissement, animation, annonce, moment, ennuyer, humour, comédie, saga, détente, divertissant, navet, requin, fan, nain, vivement, chat, recommander, conseil, vraiment, destination, spécial, sympathique, bande, voir, bond, top, agréable...

Le vocabulaire est centré sur l'effet que produit le film sur le spectateur, sur ce qu'il a ressenti pendant le visionnage du film: le registre des émotions y est fort présent (rire, adorer, décevoir, ennuyer...). Un ensemble de termes appartenant au vocabulaire de la recommandation (recommander, conseil) caractérise également ces critiques. Les critiques sont centrées sur l'expérience ressentie et partagée par le spectateur, qui le conduit à conseiller ou déconseiller le film à ses lecteurs. Étant donnée la place de l'émotion et de la recommandation dans cette deuxième classe, il est naturel que les pronoms de la $1^{\text {ère }}$ et $2^{\text {nde }}$ personne (je-vous) y soient très nettement représentés. Les critiques de la première classe empruntent au contraire un style impersonnel, qui se rapproche de la forme " savante » : attention accordée à la forme, au contenu, au média.

Il existe des liens très étroits entre la manière de rédiger et le genre des films sur lesquels portent ces critiques, comme le montre le graphique suivant (figure 5). Alors qu'en moyenne, on a $48 \%$ de critiques centrées sur l'analyse, ce taux est de $70 \%$ pour les drames et comédies dramatiques, et de $87 \%$ pour les documentaires. Inversement, pour les films d'animation, les comédies et les films d'action, le taux de critiques centrées sur la réception dépasse les $60 \%$. 
Figure 5 : Répartition des types de critiques selon les genres (\%)

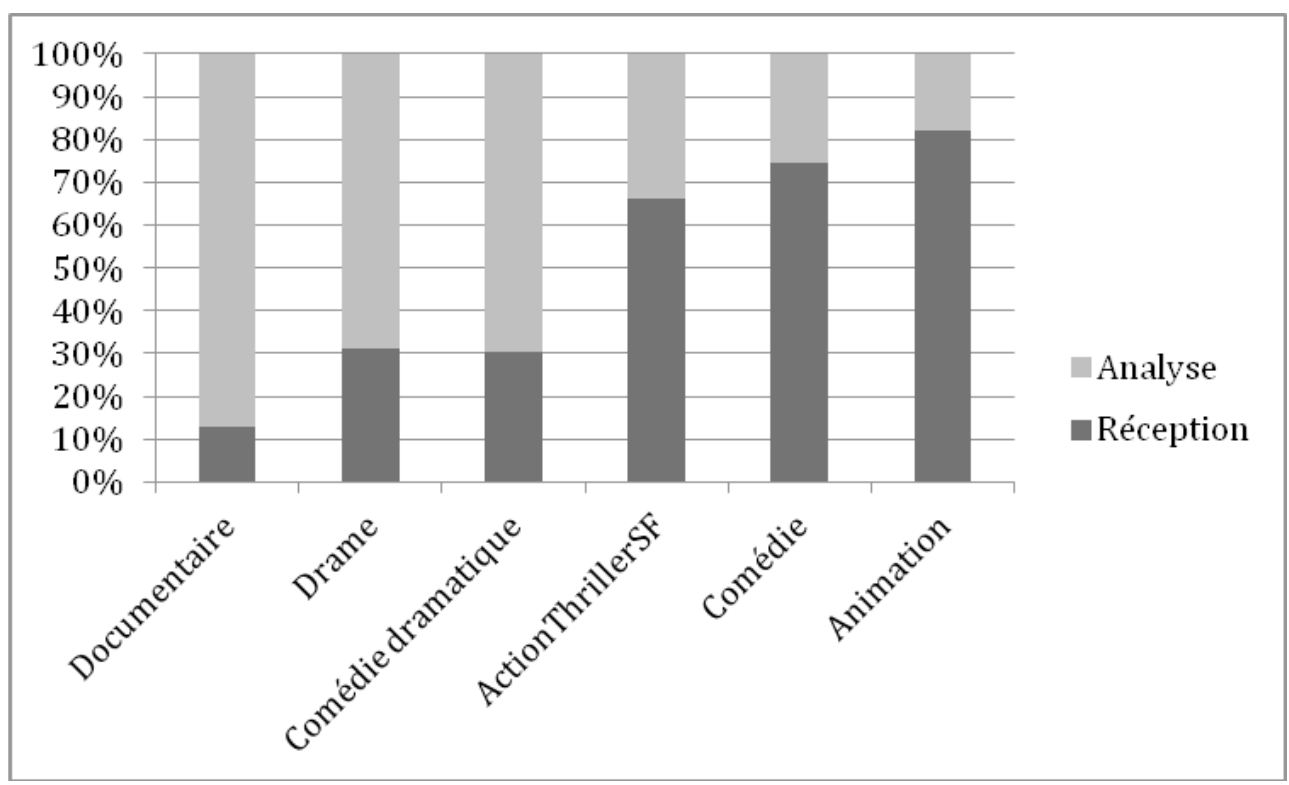

Lecture : Sur les documentaires, plus de $90 \%$ des critiques sont de type analyse et portent sur le contenu et la forme, alors que sur les films d'animation, $80 \%$ des critiques sont centrées sur la réception (émotion et recommandation).

Champ : films sortis en 2011 et encore à l'affiche fin 2011 (N : 141).

Source : site français d'information sur le cinéma («Viv@films »).

En bref, les amateurs se distinguent de la critique professionnelle par le choix des films et des genres, par la manière de noter en se positionnant contre la critique professionnelle (valoriser des films peu appréciés par les experts ou au contraire se démarquer des notations des experts) et enfin par la défense d'une forme de critique inexistante chez les professionnels (émotion et recommandation).

\section{Les amateurs, univers hétérogène}

Après avoir identifié dans les grandes lignes les différences entre la critique amateur et la critique professionnelle, nous allons maintenant examiner les tensions qui traversent la critique amateur en interne. Une dimension principale structure cet espace : elle oppose les novices aux habitués.

Nous avons stratifié notre population en fonction de l'intensité de l'exercice critique, sur la base des deux éléments disponibles, le nombre de critiques postées et l'ancienneté de l'inscription sur le site. Les novices (une seule critique depuis leur inscription sur le site) représentent un tiers des contributeurs en 2011. 47 \% d'entre eux sont inscrits sur le site depuis moins de 3 mois. Leur manière de faire des critiques est très spécifique. Ils sont plus généreux dans la notation avec une médiane de 4,5 (sur 5). Ils préfèrent les comédies et les films d'action et évitent les drames. Les critiques sont courtes avec une médiane de 30 mots. Elles privilégient l'expression du ressenti et la recommandation aux autres. C'est le cas de plus de $70 \%$ des critiques. L'analyse du film et les références au monde du cinéma y sont rares. On trouve principalement un partage impulsif de l'émotion. D'ailleurs, comme le montre le tableau 7, ces novices sont plus nombreux que la moyenne à publier la semaine de la sortie du film (47\%). Chez ces contributeurs occasionnels, le 
plaisir ressenti pendant la projection et le désir de partager semblent être le moteur principal de leur contribution, ce qui peut expliquer que les notes soient si élevées. L'inscription dans l'univers de la cinéphilie n'est pas leur préoccupation, ils prennent la plume pour inciter les autres à voir le film.

51 A l'inverse, les habitués, ceux qui ont publié sur le site plus de 11 critiques depuis leur inscription, soit $2 \%$ de nos contributeurs, ont une manière très différente d'écrire. Les notes sont moins élevées, les critiques s'allongent: deux fois plus longues en moyenne que celles des novices. Elles portent plus souvent sur des drames, moins souvent sur des comédies et des films d'action. Plus de $60 \%$ de leurs critiques sont centrées sur l'analyse de film et adoptent un ton neutre en termes de recommandation. $68 \%$ de ces contributeurs sont inscrits depuis plus d'un an et demi sur le site, contre une moyenne de $43 \% .35 \%$ sont présents depuis plus de 4 ans contre $19 \%$ en moyenne.

Entre ces deux types de participation il existe un continuum : plus on écrit de critiques, plus on évite les notes trop hautes et les critiques très courtes, plus on parle au nom de l'esthétique du film et non de ses impressions personnelles de spectateur, moins on s'intéresse à la comédie et aux films d'action.

53 Tout en haut de la hiérarchie des contributeurs, on trouve une toute petite élite aux caractéristiques particulières : les membres du Club. Numériquement, ils représentent un petit effectif (91 personnes sur les 18000 auteurs amateurs de notre corpus 2011) et ne sont même pas nécessairement les plus gros contributeurs : sur les 17 internautes qui ont produit le plus grand nombre de critiques sur nos films, seuls 5 sont membres du Club. Mais cette élite de la contribution se distingue nettement des habitués et mérite qu'on s'y arrête.

54 S'ils ne sont pas les plus gros contributeurs à l'échelle d'une année mais des contributeurs intensifs vu leur ancienneté d'inscription sur le site, ils sont des critiques d'un genre très particulier, surtout par rapport aux habitués qui, comme eux, ont l'habitude de produire un grand nombre de critiques. Tout d'abord dans $45 \%$ des cas, ils publient leur critique avant ou la semaine de la sortie du film contre $33 \%$ en moyenne. Ils sont beaucoup plus nombreux à publier des critiques avant la sortie, témoignant ainsi de leurs privilèges (présence aux avant-premières et aux projections de presse). Leurs critiques sont aussi en moyenne deux fois plus longues que celles des habitués, se rapprochant ainsi du format de la critique de presse. Ils privilégient les drames et comédies dramatiques ( $47 \%$ de leurs critiques contre $35 \%$ pour les autres) et délaissent les comédies (16\% contre $25 \%$ ). Par rapport à la critique professionnelle, ils partagent un point commun avec les autres contributeurs : une attention particulière aux films d'action, thrillers ou science-fiction. $26 \%$ de leurs critiques portent sur ce type de films contre $14 \%$ des critiques professionnelles. $84 \%$ de leurs critiques (contre $62 \%$ de celles des habitués) sont centrées sur le contenu et la forme du film, évitent l'expression émotionnelle, la présence d'un je/ vous marqueur d'une interaction avec le public et tout ce qui de près ou de loin touche à la promotion et à la recommandation. Enfin, ils évitent bien plus souvent la note maximale de 5 pour un film.

Il existe donc des différences radicales dans la manière d'exercer l'activité de critique amateur (tableau 6, 7 et 8). Les habitués ont un profil qui se distingue fortement de celui des novices, et s'il est proche de celui des membres du Club, il n'en a cependant pas toutes les propriétés. 
Tableau 6 : Caractéristiques des critiques selon le profil des contributeurs

\begin{tabular}{|l|l|l|l|}
\hline Profil des contributeurs & $\begin{array}{l}\text { Contributeurs } \\
\text { (\%) }\end{array}$ & $\begin{array}{l}\text { Critiques } \\
\text { rédigées (\%) }\end{array}$ & $\begin{array}{l}\text { Critiques publiées avant la } \\
\text { date de sortie ou pendant la } \\
\text { première semaine }\end{array}$ \\
\hline $\begin{array}{l}\text { Une seule critique publiée } \\
\text { (Novices) }\end{array}$ & $5857(32 \%)$ & $5857(15 \%)$ & $47 \%$ \\
\hline $\begin{array}{l}1 \text { ou 2 critiques sur les } \\
\text { films de } 2011\end{array}$ & $9139(50 \%)$ & $11580(29 \%)$ & $43 \%$ \\
\hline $\begin{array}{l}3 \text { à } 10 \text { critiques sur les } \\
\text { films de } 2011\end{array}$ & $2665(15 \%)$ & $12201(31 \%)$ & $40 \%$ \\
\hline $\begin{array}{l}\text { Plus de } 10 \text { critiques sur les } \\
\text { films de } 2011 \text { (Habitués) }\end{array}$ & $425(2 \%)$ & $8531(22 \%)$ & $49 \%$ \\
\hline Le Club & $91(0,5 \%)$ & $1305(3 \%)$ & $60 \%$ \\
\hline
\end{tabular}

Champ : films sortis en 2011 et encore à l'affiche fin 2011 (N : 141).

Source : site français d'information sur le cinéma («Viv@films »).

Tableau 7 : Note et longueur des critiques selon le profil des contributeurs

\begin{tabular}{|l|l|l|l|l|}
\hline & \multicolumn{2}{|l|}{ Note } & \multicolumn{2}{l|}{$\begin{array}{l}\text { Longueur de la critique (nombre de } \\
\text { mots) }\end{array}$} \\
\hline & Moyenne & Médiane & Moyenne & Médiane \\
\hline Une seule critique publiée & 3,6 & 4,5 & 52 & 30 \\
\hline $\begin{array}{l}1 \text { ou } 2 \text { critiques sur les films de } \\
2011\end{array}$ & 3,6 & 4 & 64 & 39 \\
\hline $\begin{array}{l}3 \text { à } 10 \text { critiques sur les films de } \\
2011\end{array}$ & 3,5 & 4 & 75 & 45 \\
\hline $\begin{array}{l}\text { Plus de } 10 \text { critiques sur les films } \\
\text { de } 2011\end{array}$ & 3,3 & 3,5 & 108 & 67 \\
\hline Le Club & 3,0 & 3 & 170 & 133 \\
\hline
\end{tabular}

Note : Les écarts de note et de longueur selon le profil des contributeurs sont statistiquement significatifs. (Analyse de la variance Note : F: 92.4 - $p<0.0001$; Longueur : F: $347.6-p<0.0001$ ). Champ : films sortis en 2011 et encore à l'affiche fin 2011 (N : 141).

Source : site français d'information sur le cinéma (« Viv@films »). 
Tableau 8 : Genres des films sur lesquels portent les critiques selon le profil des contributeurs (\%)

\begin{tabular}{|c|c|c|c|c|c|c|c|}
\hline & $\begin{array}{l}1 \quad \text { seule } \\
\text { critique } \\
\text { publiée }\end{array}$ & 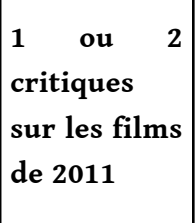 & $\begin{array}{l}3 \quad \text { à } \quad 10 \\
\text { critiques } \\
\text { sur les films } \\
\text { de } 2011\end{array}$ & 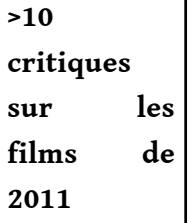 & $\begin{array}{l}\text { Le } \\
\text { Club }\end{array}$ & Total & $\begin{array}{l}\text { Critique } \\
\text { presse }\end{array}$ \\
\hline Documentaire & 1 & 1 & 1 & 1 & 3 & 1 & 10 \\
\hline Drame & 22 & 21 & 24 & 32 & 32 & 25 & 38 \\
\hline $\begin{array}{l}\text { Comédie } \\
\text { dramatique }\end{array}$ & 7 & 8 & 11 & 14 & 15 & 10 & 13 \\
\hline Comédie & 31 & 27 & 24 & 20 & 16 & 25 & 16 \\
\hline Animation & 10 & 12 & 11 & 9 & 8 & 11 & 9 \\
\hline $\begin{array}{l}\text { Action- } \\
\text { Thriller-SF }\end{array}$ & 30 & 32 & 29 & 24 & 26 & 29 & 14 \\
\hline Total (\%) & 100 & 100 & 100 & 100 & 100 & 100 & 100 \\
\hline Total effectifs & 5857 & 11580 & 12201 & 8531 & 1305 & $\begin{array}{l}39 \\
474\end{array}$ & 2324 \\
\hline
\end{tabular}

Khi2 : $1074.84 ; \mathrm{p}<0.0001$ (sans la critique presse).

Champ : films sortis en 2011 et encore à l'affiche fin 2011 ( $N$ : 141).

Source : site français d'information sur le cinéma (« Viv@films »).

Si la manière de noter, la longueur des critiques et le délai de publication sont des facteurs qui distinguent sensiblement des types de critiques amateurs, c'est la manière d'écrire sur le film qui distingue le mieux les profils de critiques: la part des critiques centrées sur le film augmente régulièrement quand les contributeurs deviennent des habitués, au détriment des critiques centrées sur la réception, témoignant de l'acquisition de codes et de normes propres au monde professionnel ${ }^{6}$. 
Figure 7 : Répartition des deux types de critiques (Analyse vs Réception) en fonction de l'intensité de la pratique (\%)

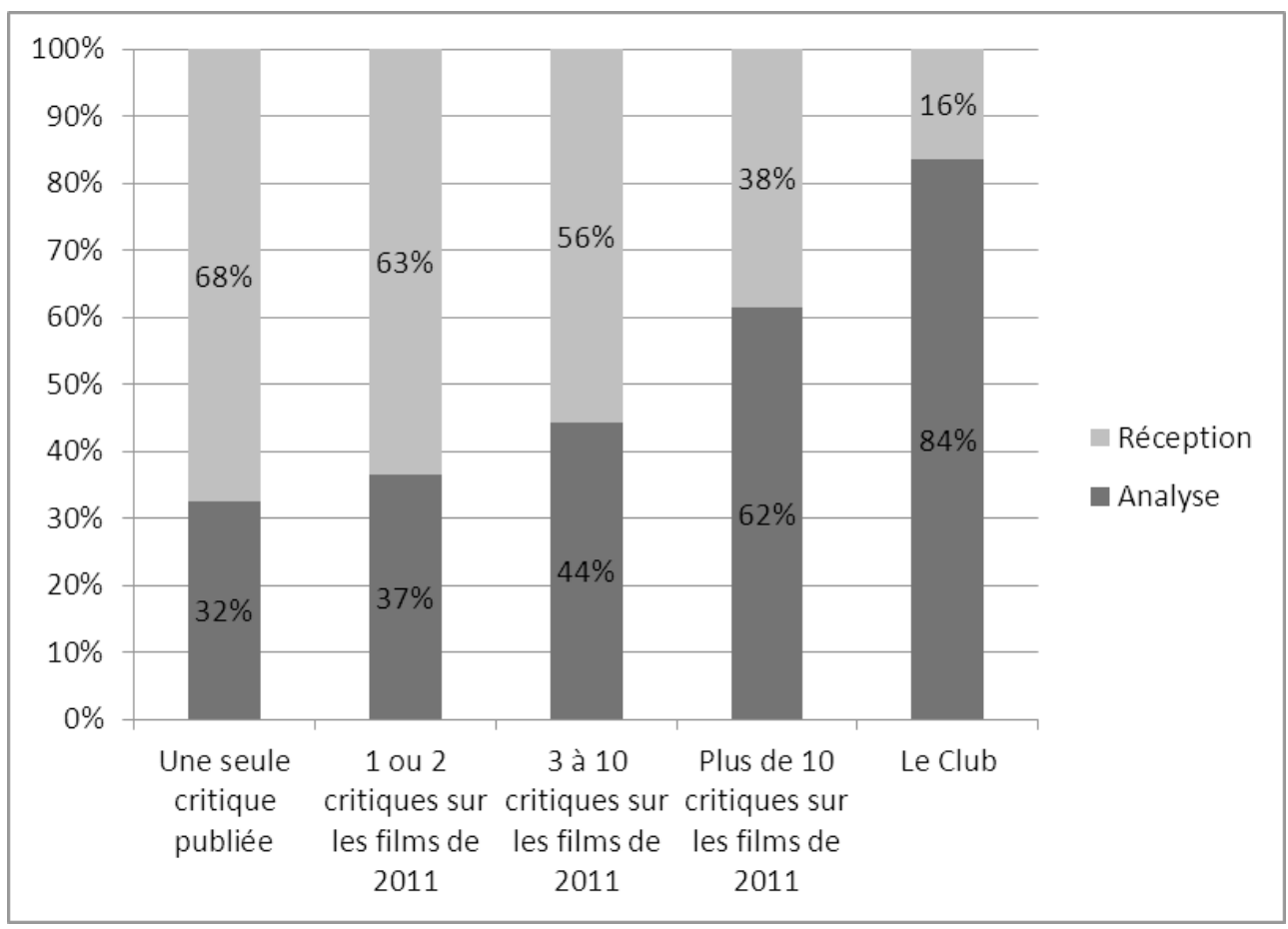

Khi2 : 1245, $\mathrm{P}<0.0001$

Champ : films sortis en 2011 et encore à l'affiche fin 2011 ( N : 141).

Source : site français d'information sur le cinéma (« Viv@films »).

\section{Conclusion}

57 Cet article a exploré la critique amateur cinéphile sous deux aspects, comme un monde qui s'oppose à la critique professionnelle, puis en interne, comme un monde structuré par les pratiques contributives.

Nous avons identifié trois différences principales avec la critique de presse, dans le choix des films, dans les manières de noter et dans l'argumentation déployée. Les amateurs ont tendance à se focaliser sur les films ayant fait un grand nombre d'entrées et sur des genres cinématographiques habituellement peu couverts par la presse comme les films d'horreur ou de science-fiction. Ils ont plus fréquemment recours à des notes extrêmes hautes comme basses - et certains d'entre eux emploient dans leur texte critique un registre fondé sur l'expression des émotions produites par le film. Comme Verboord (2014), nous avons identifié deux modèles critiques avec d'un côté des critiques esthétiques centrées sur le film (son contenu et sa forme) et de l'autre des critiques émotionnelles centrées sur sa réception (ce que le film fait au spectateur). Nous avons alors croisé les choix critiques (en termes de choix de films et de caractéristiques des contenus produits) avec les profils de contribution, mesurés par l'ancienneté d'inscription sur le site et le nombre de critiques produites, pour montrer que plus la pratique est régulière, plus la critique mobilise l'analyse esthétique et se rapproche des normes et des formats de la critique professionnelle.

Si l'on part du principe que les contributeurs les plus réguliers sont aussi ceux qui jouent le rôle le plus important dans le collectif des auteurs du fait de leur permanence et de leur 
productivité, un tel résultat tend à affaiblir l'hypothèse d'un changement des critères de l'évaluation culturelle avec la montée d'une critique amateur menée au nom de l'esthétique populaire. Cette dernière apparait être caractéristique des critiques les moins investis dans l'exercice et les moins présents dans la durée. Les conclusions de notre travail invitent donc à ne plus envisager le monde de la critique amateur en ligne comme un ensemble qui aurait la capacité d'ébranler les critères de l'évaluation des biens culturels - notamment ceux de la critique professionnelle - mais comme un rassemblement hiérarchisé de sous-groupes ayant des pratiques hétérogènes et n'ayant pas le même poids face au public des sites.

\section{BIBLIOGRAPHIE}

ALLARD Laurence (2000). « Cinéphiles, à vos claviers! Réception, public et cinéma », Réseaux, 18 (99), p. 131-168.

BEAUDOUIN Valérie \& PASQUIER Dominique (2014). « Organisation et hiérarchisation des mondes de la critique amateur cinéphile », Réseaux, 32 (183), p. 123-160.

BÉLIARD Anne-Sophie (2014). « Jeux croisés entre critique amateur et critique professionnelle dans les blogs de séries télévisées », Réseaux, 32 (183), p. 93-123

BELVAUX Bertrand \& MARTEAUX Séverine (2007). « Les recommandations d'internautes comme source d'information. Quel impact sur les entrées des films au cinéma? », Recherche et Applications en Marketing, 22 (3), pp. 65-82.

BÉRA Matthieu (2003). « Critique d'art et/ou promotion culturelle ? », Réseaux, 117, pp. 153-187. BEUSCART Jean-Samuel \& Kevin MELLET (2012). Promouvoir les œuvres culturelles - Usages et efficacité de la publicité dans les filières culturelles, Paris, La Documentation Française.

BOURDIEU Pierre (1979). La distinction. Critique sociale du jugement, Paris, Les éditions de Minuit. CRETON Laurent (2000). «Critique et promotion dans l'univers cinématographique : distinctions, conjonctions et dysphories », Entrelacs, Hors-série : Le marketing du cinéma, pp. 27-37.

DARRÉ Yann (2006). «Esquisse d'une sociologie du cinéma », Actes de la recherche en sciences sociales, 161-162, pp. 122-136.

DEBENEDETTI Stéphane (2006). «L'impact de la critique de presse sur la consommation culturelle : un essai de synthèse dans le champ cinématographique ", Recherche et applications en marketing, 21 (2), pp. 43-59.

DELlarocas Chrysanthos, ZHANG Xiaoquan (Michael) \& AWAD Neveen F. (2007). « Exploring the value of online product reviews in forecasting sales: The case of motion pictures ", Journal of Interactive Marketing, 21 (4), pp. 23-46.

DONNAT Olivier (2009). Les pratiques culturelles des Français à l'ère numérique. Enquête 2008, Paris, La Découverte. 
DUAN Wenjing, GU Bin \& WHINSTON Andrew B. (2008). « Do online reviews matter? - An empirical investigation of panel data », Decision Support Systems, 45 (4), pp. 1007-1016.

FRANÇOIS Pierre \& CHARTRAIN Valérie (2009). «Les critiques d'art contemporain : petit monde éditorial et économie de la gratuité ", Histoire et Mesure, 24 (1), pp. 3-42.

FOURNOUT Olivier, BEAUDOUIN Valérie \& FERRARESE Estelle (2014). « De l'utopie numérique à la pratique : le cas de l'annotation collaborative de films », Communication \& langages, 180, pp. 95-120.

LARCENEUX Fabrice (2001). «Critical Opinion as a Tool in the Marketing of Cultural Products: The Experiential Label », International Journal of Arts Management, 3 (2), pp. 60-70.

LEGALLOIS Dominique \& POUdAT Céline (2008). « Comment parler des livres que l'on a lus ? Discours et axiologie des avis des internautes ", Semen, 26, [en ligne], mis en ligne le 18 mars 2009. URL : http://semen.revues.org/8444

LIU Bing (2012). Sentiment Analysis and Opinion Mining, Morgan \& Claypool Publishers.

MAYZLIN Dina \& CHEVALIER Judith (2003). « The effect of word of mouth on sales : online book reviews ", Yale School of Management Working Papers.

MOULIN Raymonde (1992). L'artiste, l'institution, le marché, Paris, Flammarion.

NAULIN Sidonie (2014). « La blogosphère culinaire. Cartographie d'un espace amateur », Réseaux, 32 (183), pp. 183-216.

PASQUIER Dominique (dir.) (2014). « Evaluations profanes : le jugement en ligne », Réseaux, 32 (183).

VERBOORD Marc (2010). «The Legitimacy of Book Critics in the Age of the Internet and Omnivorousness: Expert Critics, Internet Critics and Peer Critics in Flanders and the Netherlands ", European Sociological Review, 26 (6), pp. 623-637.

VERBOORD Marc (2014). « The impact of peer-produced criticism on cultural evaluation: A multilevel analysis of discourse employment in online and offline film reviews », New Media \& Society, 16 (6), pp. 921-940.

\section{NOTES}

1. Cette recherche a été financée par le Département des Études (DEPS) du ministère de la culture et de la communication. Nous remercions Denis Vilar pour l'extraction et la mise en forme des données, et les relecteurs anonymes pour leurs suggestions constructives.

2. En majorité, les recherches « soulignent l'existence d'un lien statistique significatif et positif, d'intensité souvent modérée entre l'évaluation de la critique et le succès du film » (Debenedetti, 2006 : 50). Cet impact positif de la critique sur le succès, se vérifie particulièrement dans le cas des œuvres offrant peu de signaux de qualité attractifs pour le grand public et dont les budgets marketing sont peu élevés. Les blockbusters affichant une star à l'affiche seraient en quelque sorte « critic proof» note Debenedetti.

3. Voir Béra (2003), Moulin (1992) et François \& Chartrain (2009) pour la critique d'art.

4. Au départ, la sélection des membres du Club s'est faite pour moitié auprès des blogueurs cinéma influents repérés sur internet par les responsables du site, et pour moitié auprès des contributeurs les plus actifs sur le site. Dans les entretiens, les professionnels assument une certaine part de subjectivité dans leurs choix et disent chercher à représenter des profils assez 
différents. En tout cas, le volume de contribution semble peser moins que les qualités d'écriture et l'érudition cinéphile.

5. Éviter les critiques de moins de 50 caractères (contrainte majeure inscrite dans le système, puisque les critiques de moins de 50 caractères ne sont pas validées) et de plus de 1000 caractères et privilégier un calibrage entre 200 et 500 caractères. La charte souligne l'importance donnée à l'argumentation ("pourquoi vous avez aimé ou pas le film»). Elle insiste sur le fait qu'il faut éviter le dialogue dans les critiques, c'est un jugement individuel qui s'exprime («vos critiques ne doivent en aucun cas réagir aux avis d'autres internautes ou se référer à de précédentes critiques »). En cela, le modèle de la critique se différencie radicalement des échanges dans les forums. Enfin, des consignes sont données pour ne pas révéler des éléments importants du film : la partie du texte concernée doit être marquée par «spoiler » et devient alors invisible.

6. Dans le cadre de l'expérimentation d'un outil d'annotation de films, testé par une quinzaine de personnes diplômées du supérieur, nous avions observé que le registre émotionnel présent dans les premières annotations avait fini par disparaitre au profit d'annotations critiques relevant du modèle de la critique scolaire ou professionnelle. L'expression des émotions était devenue peu à peu illégitime (Fournout et al., 2014).

\section{RÉSUMÉS}

La critique amateur en ligne propose-t-elle une approche des films différente de celle de la critique professionnelle ? La question est posée à partir d'un corpus de 2300 critiques professionnelles et 40000 critiques amateurs postées en ligne sur un site français d'information sur le cinéma. Les auteurs identifient trois différences principales: dans le choix des films critiqués, dans les manières de les noter et dans la mobilisation d'un registre émotionnel par une partie des internautes. Mais lorsqu'on croise les choix critiques (en termes de choix de films et de caractéristiques des contenus produits) avec les profils de contribution, mesurés par l'ancienneté d'inscription sur le site et le nombre de critiques produites, il apparaît que plus la pratique est régulière, plus la critique mobilise l'analyse esthétique et se rapproche des normes et des formats de la critique professionnelle.

Do amateurs have a different approach to critique than professionals? On the basis of analysis of 2,300 professional and 40,000 amateur reviews posted on a French web based platform providing information on cinema, the authors identify three main differences: in the choice of films criticized, in the ratings given, and in a reviewing model centered on reception and emotions used by part of the amateurs. They also show that the profiles of contributors (number of reviews posted, length of time of subscription to the site) is related to the type of reviews posted (choice of films, models of argumentation): the more often an author post reviews, the closer the critique will be to the norms and formats of a professional critique.

\section{INDEX}

Keywords : amateur critique, professional critique, cinema, online contribution, evaluation Mots-clés : critique amateur, critique professionnelle, cinéma, contribution en ligne, évaluation culturelle 


\section{AUTEURS}

\section{VALÉRIE BEAUDOUIN}

Télécom Paris-Tech, Département SES

DOMINIQUE PASQUIER

CNRS, Télécom Paris-Tech 